\title{
Quantum memory for Rindler supertranslations
}

\author{
Sanved Kolekar ${ }^{1,2,3, *}$ and Jorma Louko, ${ }^{1, \dagger}$ \\ ${ }^{1}$ School of Mathematical Sciences, University of Nottingham, Nottingham NG7 2RD, United Kingdom \\ ${ }^{2}$ CPT, Aix Marseille Université, Université de Toulon, CNRS, UMR 7332, 13288 Marseille, France \\ ${ }^{3} U M-D A E$ Centre for Excellence in Basic Sciences, Mumbai 400098, India
}

(Received 10 October 2017; published 16 April 2018)

\begin{abstract}
The Rindler horizon in Minkowski spacetime can be implanted with supertranslation hair by a matter shock wave without planar symmetry, and the hair is observable as a supertranslation memory on the Rindler family of uniformly linearly accelerated observers. We show that this classical memory is accompanied by a supertranslation quantum memory that modulates the entanglement between the opposing Rindler wedges in quantum field theory. A corresponding phenomenon across a black hole horizon may play a role in Hawking, Perry, and Strominger's proposal for supertranslations to provide a solution to the black hole information paradox.
\end{abstract}

DOI: 10.1103/PhysRevD.97.085012

\section{INTRODUCTION}

In the longstanding pursuit to predict the fate of an evaporating black hole, a recent development is the suggestion by Hawking, Perry, and Strominger [1-3] that significant quantum correlations may be encoded in "soft" degrees of freedom, associated with vanishing energy in a particle description and a diffeomorphism in a geometric description, but nevertheless carrying nontrivial dynamics due to the global boundary conditions. Such degrees of freedom exist already in Minkowski spacetime electrodynamics [4], and in the gravitational case these degrees of freedom are associated with supertranslations in the BondiMetzner-Sachs (BMS) group of asymptotic isometries at the infinity [5-10]. Contributions to the ongoing debate include $[11,12]$.

In this paper we analyze the correspondence between quantum correlations and classical supertranslations in the simplified setting where a stationary black hole horizon is replaced by the Rindler horizon, the Killing horizon of a boost Killing vector in Minkowski spacetime. This simplification has a long pedigree [13-15], avoiding complications due to spacetime curvature but maintaining a bifurcate Killing horizon as a central piece of input in the quantum field theory $[16,17]$. We shall analyze how the quantum correlations across the Rindler horizon change

\footnotetext{
sanved.kolekar@cbs.ac.in

jorma.louko@nottingham.ac.uk
}

Published by the American Physical Society under the terms of the Creative Commons Attribution 4.0 International license. Further distribution of this work must maintain attribution to the author(s) and the published article's title, journal citation, and DOI. Funded by SCOAP. when the horizon is implanted with classical supertranslation hair.

Recall that the Schwarzschild black hole can be implanted with supertranslation hair by letting a spherically asymmetric shock wave fall into the hole [2]. This classical hair is observable in the gravitational memory that affects the separation of geodesic observers at the asymptotic infinity [18], and in quantum field theory it is expected to be accompanied by correlations in the outgoing Hawking quanta.

For a Rindler horizon, the notion of supertranslation hair has been characterized in [19-22] (for a related discussion see [23]). It was shown in [24] that the Rindler horizon can be implanted with supertranslation hair by letting a shock wave without planar symmetry fall across the horizon, and this hair is classically observable in a memory on the Rindler family of uniformly linearly accelerated observers. We shall show that the classical Rindler supertranslation memory is accompanied by a Rindler supertranslation quantum memory, and we analyze how this memory modulates the entanglement between the opposing Rindler wedges.

We work with a massless scalar field in $3+1$ spacetime dimensions. The core results are given in terms of a Bogoliubov transformation between a presupertranslation region and a postsupertranslation region, demonstrating that both the alpha-coefficients and beta-coefficients are nontrivial, so that the supertranslation induces both particle creation and mode mixing. The entanglement is analyzed within a truncation to finitely many field modes, and using negativity as the entanglement monotone. We identify subsystems in which entanglement is degraded and subsystems in which the entanglement is generated, and this identification appears reasonably robust against the input used in the truncation.

We anticipate that a similar analysis can be carried out for supertranslations implanted on a Schwarzschild black 
hole as in [2], and that the results will help to clarify the role of black hole supertranslations in the solution to the black hole information paradox.

We begin in Sec. II with a recap of the classical Rindler supertranslation memory [24]. The quantum memory is found in Sec. III, and the entanglement consequences are analyzed in Sec. IV. Section V gives a summary and brief concluding remarks. Appendix A gives the derivation of an integral identity used in the main text, and Appendix B recalls key features of negativity as an entanglement monotone.

The Minkowski metric is taken to have the mostly plus sign, and Roman indices run over all spacetime indices. Complex conjugate is denoted by an asterisk and Hermitian conjugate by a dagger.

\section{RECAP: CLASSICAL MEMORY FOR RINDLER SUPERTRANSLATIONS}

In this section we recall relevant properties of the Rindler spacetime and the classical Rindler supertranslation memory found in [24], establishing the notation that will be used in the quantum field theory analysis in Sec. III.

The spacetime consists of two subsets of four-dimensional Minkowski spacetime joined together along a null shell as shown in Fig. 1. In the past of the shell we write the metric in the Minkowski null coordinates $(U, V, x, y)$ as

$$
d s^{2}=-d U d V+\delta_{A B} d x^{A} d x^{B},
$$

where the uppercase Latin indices take values in $\{x, y\}$ and the shell is at $V=V_{0}>0$. In the region $0<V<V_{0}$, we introduce advanced Bondi-type coordinates $(v, r, x, y)$ in which the metric reads

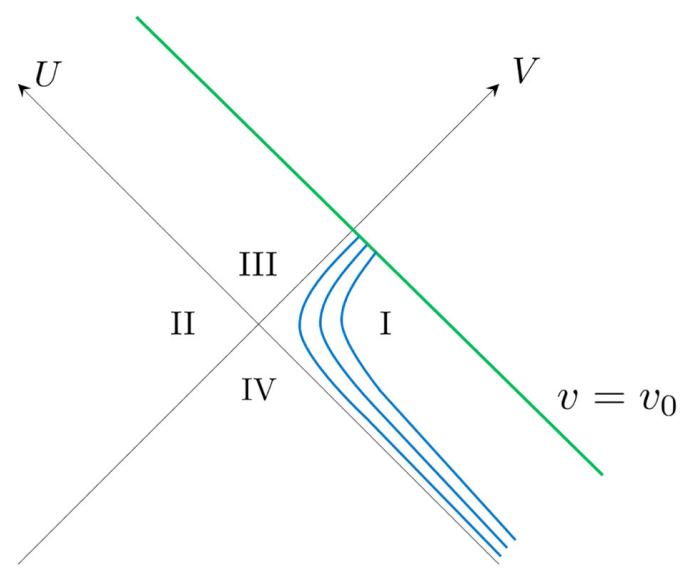

FIG. 1. A null shell in the Rindler spacetime, with the transverasse dimensions suppressed. In the past of the shell the metric is given by (2.1), and the shell is at $V=V_{0}>0$. The four Rindler quadrants are labeled in the figure by I, II, III, and IV, and selected orbits of the boost Killing vector $\bar{\xi}$ (2.3) are shown in quadrant I. The coordinates in (2.5) cover both the future of the shell and the region $0<V<V_{0}$ of the past of the shell, and in these coordinates the shell is at $v=v_{0}$.

$$
d s^{2}=-2 \kappa r d v^{2}+2 d v d r+\delta_{A B} d x^{A} d x^{B},
$$

where $\kappa$ is a positive constant of dimension inverse length, $-\infty<r<\infty,-\infty<v<v_{0}$, and the shell is at $v=v_{0}$. In terms of the four Rindler quadrants shown in Fig. 1, $r>0$ is in region I, $r<0$ is in region III, and $r=0$ is on the Rindler horizon, $U=0$. Curves of constant $r, x$, and $y$ are orbits of the boost Killing vector

$$
\bar{\xi}=-U \partial_{U}+V \partial_{V}=\kappa^{-1} \partial_{v} .
$$

Selected orbits of $\bar{\xi}$ in region I are shown in the figure.

In the future of the shell, $v>v_{0}$, we take the metric to be related to (2.2) by the diffeomorphism that is generated by the vector field

$$
\Xi^{a}=\kappa^{-1}\left[f(x, y), 0,-r \partial^{A} f(x, y)\right],
$$

where $f$ is an arbitrary function of the transverse coordinates. Working to linear order in $f$, the metric for $-\infty<v<\infty$ thus reads

$$
\begin{aligned}
d s^{2}= & -2 \kappa r d v^{2}+2 d v d r+4 r h\left(v-v_{0}\right) \partial_{A} f d v d x^{A} \\
& +\left(\delta_{A B}+2 \kappa^{-1} r h\left(v-v_{0}\right) \partial_{A} \partial_{B} f\right) d x^{A} d x^{B},
\end{aligned}
$$

where $h\left(v-v_{0}\right)$ is the Heaviside step function.

While $\partial_{v}$ is not a Killing vector of (2.5) at $v=v_{0}$, it is a Killing vector individually both for $v<v_{0}$ and for $v>v_{0}$, and in each region it generates a pure boost: for $v<v_{0}$ this holds by (2.3), and for $v>v_{0}$ this holds because $\partial_{v}$ commutes with $\Xi$ (2.4). As $g_{r r}=0$ and $g_{v r}=2$, the coordinates in (2.5) may be regarded as a Rindler counterpart of Bondi-type coordinates, and the coordinates preserve the structure of the Rindler horizon for all $v$ in the sense that $g_{v v}=\mathcal{O}(r)$ and $g_{A v}=\mathcal{O}(r)$. $\Xi$ may hence be regarded as a Rindler version of a supertranslation vector field [19-22], and it follows that the shell imparts a Rindler supertranslation charge on the spacetime. The linearized stress-energy tensor vanishes for $v \neq v_{0}$ by construction, while at $v=v_{0}$ the stress-energy tensor is nonvanishing for generic $f$, involving not just the Dirac delta but also the derivative of the Dirac delta [24].

It was shown in [24] that the supertranslation charge imparted by the shell is detectable as a classical memory on a family of observers who prior to the shell are uniformly linearly accelerated, along the orbits of the boost Killing vector $\bar{\xi}$ (2.3). Assuming that each of these observers maintains their uniform linear acceleration on crossing the shell, as characterized at the shell by the appropriate local notion of acceleration in curved spacetime, the observers follow after the shell orbits of boost Killing vectors that differ from trajectory to trajectory, and the trajectorydependence carries a memory of the planar inhomogeneity of the shell. In the rest of the paper we show that an accompanying memory exists also for a quantum field. 


\section{QUANTUM MEMORY FOR RINDLER SUPERTRANSLATIONS}

We now turn to a real massless Klein-Gordon field in the $r>0$ part of the shock wave spacetime (2.5). Geometrically, $r>0$ means that we only consider the right quadrant of the spacetime as shown in Fig. 1, but therein both the preshell region $v<v_{0}$ and the postshell region $v>v_{0}$.

\section{A. Classical field}

Working to linear order in $f$, the Klein-Gordon field equation, $\nabla_{a} \nabla^{a} \phi=0$, takes the form

$$
\begin{aligned}
0= & 2 \kappa r \partial_{r}^{2} \phi+2 \partial_{r} \partial_{v} \phi+\partial_{x}^{2} \phi+\partial_{y}^{2} \phi+2 \kappa \partial_{r} \phi \\
& -4 r h\left(\partial^{A} f\right) \partial_{r} \partial_{A} \phi-2 \kappa^{-1} r h\left(\partial^{A} \partial^{B} f\right) \partial_{A} \partial_{B} \phi \\
& -2 h\left(\partial^{A} f\right) \partial_{A} \phi-2 \kappa^{-1} r h\left(\partial^{A} \partial_{B} \partial^{B} f\right) \partial_{A} \phi \\
& +\kappa^{-1} h\left(\partial_{B} \partial^{B} f\right) \partial_{v} \phi+\kappa^{-1} r h^{\prime}\left(\partial_{B} \partial^{B} f\right) \partial_{r} \phi
\end{aligned}
$$

where the derivatives in parentheses act only within the parentheses. We consider this equation first individually for $v<v_{0}$ and $v>v_{0}$, and then match the solutions at $v=v_{0}$.

For $v<v_{0}$, the metric is given by (2.2), the terms proportional to $f$ in (3.1) vanish, and (3.1) reduces to

$$
0=2 \kappa r \partial_{r}^{2} \phi+2 \partial_{r} \partial_{v} \phi+\partial_{x}^{2} \phi+\partial_{y}^{2} \phi+2 \kappa \partial_{r} \phi .
$$

Separating (3.2) with the ansatz

$$
\phi=e^{-i \omega v} \phi_{r}(r) e^{i k_{A} x^{A}},
$$

where $k_{x}, k_{y}, \omega \in \mathbb{R}$, we find that $\phi_{r}(r)$ satisfies

$$
2 \kappa r \phi_{r}^{\prime \prime}+2(\kappa-i \omega) \phi_{r}^{\prime}-k^{2} \phi_{r}=0
$$

where $k=\sqrt{k_{x}^{2}+k_{y}^{2}}$. Assuming $\left(k_{x}, k_{y}\right) \neq(0,0)$, so that $k>0$, (3.4) can be solved in terms of modified Bessel functions [25], and the solution that does not diverge at $r \rightarrow \infty$ is

$$
\phi_{r}=N_{1}\left(\frac{2 k \sqrt{r}}{\sqrt{2 \kappa}}\right)^{i \omega / \kappa} K_{i \omega / \kappa}\left(\frac{2 k \sqrt{r}}{\sqrt{2 \kappa}}\right),
$$

where $N_{1}$ is a normalization factor. Solutions that are of positive frequency with respect to the boost Killing vector $\bar{\xi}$ (2.3) are those with $\omega>0$.

To fix the normalization, we define the Klein-Gordon inner product on the null surfaces of constant $v$, as in $[26,27]$. Using (2.2), the formula for the inner product becomes

$$
\left\langle W_{1}, W_{2}\right\rangle=-i \int_{0}^{\infty} d r \int_{\mathbb{R}^{2}} d x d y\left(W_{1} \partial_{r} W_{2}^{*}-W_{2}^{*} \partial_{r} W_{1}\right) .
$$

We have verified that this inner product is conserved, and it agrees with the inner product on surfaces that are deformed near $r=0$ to be spacelike and hit the Rindler horizon bifurcation point $(U, V)=(0,0)$. For a complete set of solutions that are positive frequency with respect to $\bar{\xi}$, we hence choose

$$
\begin{aligned}
\phi_{\omega, \mathbf{k}}^{0}= & \sqrt{\frac{\sinh (\pi \omega / \kappa)}{4 \pi^{4} \kappa}} e^{-i \omega v}\left(\frac{2 k \sqrt{r}}{\sqrt{2 \kappa}}\right)^{i \omega / \kappa} \\
& \times K_{i \omega / \kappa}\left(\frac{2 k \sqrt{r}}{\sqrt{2 \kappa}}\right) e^{i k_{A} x^{4}},
\end{aligned}
$$

where $\omega>0$ and $\mathbf{k}=\left(k_{x}, k_{y}\right) \in \mathbb{R}^{2} \backslash\{(0,0)\}$. The inner products are

$$
\begin{aligned}
& \left\langle\phi_{\omega, \mathbf{k}}^{0}, \phi_{\omega^{\prime}, \mathbf{k}^{\prime}}^{0}\right\rangle=\delta\left(\omega-\omega^{\prime}\right) \delta^{2}\left(\mathbf{k}-\mathbf{k}^{\prime}\right), \\
& \left\langle\phi_{\omega, \mathbf{k}}^{0 *}, \phi_{\omega^{\prime}, \mathbf{k}^{\prime}}^{0 *}\right\rangle=-\delta\left(\omega-\omega^{\prime}\right) \delta^{2}\left(\mathbf{k}-\mathbf{k}^{\prime}\right), \\
& \left\langle\phi_{\omega, \mathbf{k}}^{0}, \phi_{\omega^{\prime}, \mathbf{k}^{\prime}}^{0 *}\right\rangle=0 .
\end{aligned}
$$

For $v>v_{0}$, we have $h\left(v-v_{0}\right)=1$, and the terms proportional to $f$ in (3.1) do contribute. However, since the $v>v_{0}$ region of (2.5) is obtained from (2.2) by a diffeomorphism generated by the Rindler supertranslation vector field $\Xi$ (2.4), and since we are working to linear order in $f$, a complete set of mode solutions that are of positive frequency with respect to $\partial_{v}$ is

$$
\begin{aligned}
\phi_{\omega, \mathbf{k}}^{1} & =\left(1-\Xi^{a} \partial_{a}\right) \phi_{\omega, \mathbf{k}}^{0} \\
& =\left(1+i \kappa^{-1} \omega f+i \kappa^{-1} r k_{A} \partial^{A} f\right) \phi_{\omega, \mathbf{k}}^{0},
\end{aligned}
$$

where again $\omega>0$ and $\mathbf{k}=\left(k_{x}, k_{y}\right) \in \mathbb{R}^{2} \backslash\{(0,0)\}$. The Klein-Gordon inner product formula can be written down by applying the Rindler supertranslation diffeomorphism to (3.6), and the diffeomorphism construction guarantees that the inner products are

$$
\begin{aligned}
& \left\langle\phi_{\omega, \mathbf{k}}^{1}, \phi_{\omega^{\prime}, \mathbf{k}^{\prime}}^{1}\right\rangle=\delta\left(\omega-\omega^{\prime}\right) \delta^{2}\left(\mathbf{k}-\mathbf{k}^{\prime}\right), \\
& \left\langle\phi_{\omega, \mathbf{k}}^{1 *}, \phi_{\omega^{\prime}, \mathbf{k}^{\prime}}^{1 *}\right\rangle=-\delta\left(\omega-\omega^{\prime}\right) \delta^{2}\left(\mathbf{k}-\mathbf{k}^{\prime}\right), \\
& \left\langle\phi_{\omega, \mathbf{k}}^{1}, \phi_{\omega^{\prime}, \mathbf{k}^{\prime}}^{1 *}\right\rangle=0 .
\end{aligned}
$$

Now, consider the matching at $v=v_{0}$. We look for a solution to the linearized Klein-Gordon equation (3.1) as $\phi=\phi_{0}+\phi_{1}+\mathcal{O}\left(f^{2}\right)$, where $\phi_{0}$ has order $f^{0}$ and $\phi_{1}$ has order $f$. Matching terms order by order shows that $\phi_{0}$ satisfies (3.2) and $\phi_{1}$ satisfies

$$
\begin{aligned}
0= & 2 \kappa r \partial_{r}^{2} \phi_{1}+2 \partial_{r} \partial_{v} \phi_{1}+\partial_{x}^{2} \phi_{1}+\partial_{y}^{2} \phi_{1}+2 \kappa \partial_{r} \phi_{1} \\
& -4 r h\left(\partial^{A} f\right) \partial_{r} \partial_{A} \phi_{0}-2 \kappa^{-1} r h\left(\partial^{A} \partial^{B} f\right) \partial_{A} \partial_{B} \phi_{0} \\
& -2 h\left(\partial^{A} f\right) \partial_{A} \phi_{0}-2 \kappa^{-1} r h\left(\partial^{A} \partial_{B} \partial^{B} f\right) \partial_{A} \phi_{0} \\
& +\kappa^{-1} h\left(\partial_{B} \partial^{B} f\right) \partial_{v} \phi_{0}+\kappa^{-1} r h^{\prime}\left(\partial_{B} \partial^{B} f\right) \partial_{r} \phi_{0} .
\end{aligned}
$$

Assuming $\phi_{0}$ to be smooth across $v=v_{0}$, the terms involving $\partial_{r} \partial_{v} \phi_{1}$ and $h^{\prime}$ in (3.11) show that $\partial_{r} \phi_{1}$ has at $v=v_{0}$ a discontinuity, and the matching condition reads 


$$
2\left[\partial_{r} \phi_{1}\right]_{v_{0-}}^{v_{0+}}=-\left.\kappa^{-1} r\left(\partial_{B} \partial^{B} f\right) \partial_{r} \phi_{0}\right|_{v_{0}} .
$$

Assuming that $r \partial_{r} \phi_{0}$ is integrable at $r \rightarrow \infty$ and $\left[\phi_{1}\right]_{v_{0-}}^{v_{0+}} \rightarrow 0$ as $r \rightarrow \infty$, which will hold for the functions below, we may integrate (3.12) to

$$
2\left[\phi_{1}\right]_{v_{0-}}^{v_{0+}}=\left.\kappa^{-1}\left(\partial_{B} \partial^{B} f\right) \int_{r}^{\infty} d r r \partial_{r} \phi_{0}\right|_{v_{0}} .
$$

Consider hence the solution $\hat{\phi}_{\omega, \mathbf{k}}$ that is equal to $\phi_{\omega, \mathbf{k}}^{0}$ at $v<v_{0}$. Expanding this solution at $v>v_{0}$ in the basis $\left\{\phi_{\omega, \mathbf{k}}^{1}\right\}$, we write

$$
\hat{\phi}_{\omega, \mathbf{k}}= \begin{cases}\phi_{\omega, \mathbf{k}}^{0} & \text { for } v<v_{0} \\ \int_{0}^{\infty} d \omega^{\prime} \int d^{2} \mathbf{k}^{\prime}\left(\alpha_{\omega, \mathbf{k} ; \omega^{\prime}, \mathbf{k}^{\prime}} \phi_{\omega^{\prime}, \mathbf{k}^{\prime}}^{1}+\beta_{\omega, \mathbf{k} ; \omega^{\prime}, \mathbf{k}^{\prime}} \phi_{\omega^{\prime}, \mathbf{k}^{\prime}}^{1 *}\right) & \text { for } v>v_{0},\end{cases}
$$

where the $\alpha \mathrm{s}$ and $\beta \mathrm{s}$ are the Bogoliubov coefficients between the $\left\{\phi_{\omega, \mathbf{k}}^{0}\right\}$ basis and the $\left\{\phi_{\omega, \mathbf{k}}^{1}\right\}$ basis [16]. Using 3.9)) and (3.12), we find

$$
\begin{aligned}
\alpha_{\omega, \mathbf{k} ; \omega^{\prime}, \mathbf{k}^{\prime}}= & \delta\left(\omega-\omega^{\prime}\right) \delta^{2}\left(\mathbf{k}-\mathbf{k}^{\prime}\right)+\alpha_{\omega, \mathbf{k} ; \omega^{\prime}, \mathbf{k}^{\prime}}^{(1)} \\
& +\mathcal{O}\left(f^{2}\right), \\
\beta_{\omega, \mathbf{k} ; \omega^{\prime}, \mathbf{k}^{\prime}}= & \beta_{\omega, \mathbf{k} ; \omega^{\prime}, \mathbf{k}^{\prime}}^{(1)}+\mathcal{O}\left(f^{2}\right),
\end{aligned}
$$

where the condition determining $\alpha^{(1)}$ and $\beta^{(1)}$ is that the equation

$$
\begin{gathered}
2 \int_{0}^{\infty} d \omega^{\prime} \int d^{2} \mathbf{k}^{\prime}\left(\alpha_{\omega, \mathbf{k} ; \omega^{\prime}, \mathbf{k}^{\prime}}^{(1)} \phi_{\omega^{\prime}, \mathbf{k}^{\prime}}^{0}+\beta_{\omega, \mathbf{k} ; \omega^{\prime}, \mathbf{k}^{\prime}}^{(1)} \phi_{\omega^{\prime}, \mathbf{k}^{\prime}}^{0 *}\right) \\
=\kappa^{-1}\left(\partial_{B} \partial^{B} f\right) \int_{r}^{\infty} d r r \partial_{r} \phi_{\omega, \mathbf{k}}^{0}+2 \Xi^{a} \partial_{a} \phi_{\omega, \mathbf{k}}^{0}
\end{gathered}
$$

holds on the surface $v=v_{0}$. Evaluating $\left\langle\cdot, \phi_{\omega^{\prime}, \mathbf{k}^{\prime}}^{0}\right\rangle_{v_{0}}$ and $\left\langle\cdot, \phi_{\omega^{\prime}, \mathbf{k}^{\prime}}^{0 *}\right\rangle_{v_{0}}$ on both sides of (3.16), where $\langle\cdot, \cdot\rangle_{v_{0}}$ stands for the inner product (3.6) evaluated on the $v=v_{0}$ surface, and using (3.8), we hence obtain

$$
\begin{aligned}
\alpha_{\omega, \mathbf{k} ; \omega^{\prime}, \mathbf{k}^{\prime}}^{(1)}= & \left\langle\Xi^{a} \partial_{a} \phi_{\omega, \mathbf{k}}^{0}, \phi_{\omega^{\prime}, \mathbf{k}^{\prime}}^{0}\right\rangle_{v_{0}} \\
& +(2 \kappa)^{-1}\left\langle\left(\partial_{B} \partial^{B} f\right) \int_{r}^{\infty} d r r \partial_{r} \phi_{\omega, \mathbf{k}}^{0}, \phi_{\omega^{\prime}, \mathbf{k}^{\prime}}^{0}\right\rangle_{v_{0}}, \\
\beta_{\omega, \mathbf{k} ; \omega^{\prime}, \mathbf{k}^{\prime}}^{(1)}= & -\left\langle\Xi^{a} \partial_{a} \phi_{\omega, \mathbf{k}}^{0}, \phi_{\omega^{\prime}, \mathbf{k}^{\prime}}^{0 *}\right\rangle_{v_{0}} \\
& -(2 \kappa)^{-1}\left\langle\left(\partial_{B} \partial^{B} f\right) \int_{r}^{\infty} d r r \partial_{r} \phi_{\omega, \mathbf{k}}^{0}, \phi_{\omega^{\prime}, \mathbf{k}^{\prime}}^{0 *}\right\rangle_{v_{0}} .
\end{aligned}
$$

Writing

$$
\Xi^{a} \partial_{a} \phi_{\omega, \mathbf{k}}^{0}=-i \kappa^{-1} \omega f \phi_{\omega, \mathbf{k}}^{0}-i \kappa^{-1} r k_{A} \partial^{A} f \phi_{\omega, \mathbf{k}}^{0},
$$

we can evaluate (3.17) using formula 6.576.4 in [28] and the integral identity that we give in Appendix A. We find

$$
\begin{aligned}
\alpha_{\omega, \mathbf{k} ; \omega^{\prime}, \mathbf{k}^{\prime}}^{(1)}= & \frac{i \omega \tilde{f}(\tilde{\mathbf{k}})}{4 \pi^{2} \kappa}\left(\frac{k}{k^{\prime}}\right)^{i \omega / \kappa}\left[1+\frac{i \omega}{2 \kappa}\left(1-\frac{k^{2}}{k^{\prime 2}}\right){ }_{2} F_{1}\left(1+\frac{i \omega}{\kappa}, 1 ; 2 ; 1-\frac{k^{2}}{k^{\prime 2}}\right)\right] \delta\left(\omega-\omega^{\prime}\right) \\
& -\frac{i \omega \tilde{f}(\tilde{\mathbf{k}}) \sqrt{\sinh (\pi \omega / \kappa) \sinh \left(\pi \omega^{\prime} / \kappa\right)}}{32 \pi^{2} \kappa^{3} \sinh \left(\pi\left(\omega+\omega^{\prime}\right) /(2 \kappa)\right)} P\left(\frac{\omega+\omega^{\prime}}{\sinh \left(\pi\left(\omega-\omega^{\prime}\right) /(2 \kappa)\right)}\right) \\
& \times\left(\frac{k}{k^{\prime}}\right)^{i \omega^{\prime} / \kappa}\left(1-\frac{k^{2}}{k^{\prime 2}}\right){ }_{2} F_{1}\left(1+\frac{i\left(\omega+\omega^{\prime}\right)}{2 \kappa}, 1+\frac{i\left(\omega^{\prime}-\omega\right)}{2 \kappa} ; 2 ; 1-\frac{k^{2}}{k^{\prime 2}}\right) \\
& -\frac{i \tilde{f}(\tilde{\mathbf{k}}) e^{-i\left(\omega-\omega^{\prime}\right) v_{0}} \sqrt{\sinh (\pi \omega / \kappa) \sinh \left(\pi \omega^{\prime} / \kappa\right)}}{8 \pi^{4} \kappa}\left(\frac{k}{\sqrt{2} \kappa}\right)^{-i \omega / \kappa}\left(\frac{k^{\prime}}{\sqrt{2} \kappa}\right)^{i \omega^{\prime} / \kappa} \\
& \times \Gamma\left(1+\frac{i \omega}{\kappa}\right) \Gamma\left(1-\frac{i \omega^{\prime}}{\kappa}\right) \Gamma\left(1+\frac{i \omega}{\kappa}-\frac{i \omega^{\prime}}{\kappa}\right) \\
& \times\left[\frac{\tilde{k}^{2}}{k^{2}}\left(1-\frac{i \omega^{\prime}}{\kappa}\right){ }_{2} F_{1}\left(2,1+\frac{i \omega}{\kappa} ; 3+\frac{i \omega}{\kappa}-\frac{i \omega^{\prime}}{\kappa} ; 1-\left(\frac{k^{\prime}}{k}\right)^{2}\right)\right. \\
& \left.+\frac{4 k_{A} \tilde{k}^{A}}{\left(k^{\prime}\right)^{2}}\left(1+\frac{i \omega}{\kappa}\right)_{2} F_{1}\left(2,1-\frac{i \omega^{\prime}}{\kappa} ; 3+\frac{i \omega}{\kappa}-\frac{i \omega^{\prime}}{\kappa} ; 1-\left(\frac{k}{k^{\prime}}\right)^{2}\right)\right]
\end{aligned}
$$




$$
\begin{aligned}
\beta_{\omega, \mathbf{k} ; \omega^{\prime}, \mathbf{k}^{\prime}}^{(1)}= & \frac{i \omega \tilde{f}\left(\tilde{\mathbf{k}}_{+}\right) \sqrt{\sinh (\pi \omega / \kappa) \sinh \left(\pi \omega^{\prime} / \kappa\right)}}{32 \pi^{2} \kappa^{3} \sinh \left(\pi\left(\omega+\omega^{\prime}\right) /(2 \kappa)\right)}\left(\frac{\omega-\omega^{\prime}}{\sinh \left(\pi\left(\omega-\omega^{\prime}\right) /(2 \kappa)\right)}\right) \\
& \times\left(\frac{k}{k^{\prime}}\right)^{i \omega^{\prime} / \kappa}\left(1-\frac{k^{2}}{k^{\prime 2}}\right)_{2} F_{1}\left(1+\frac{i\left(\omega+\omega^{\prime}\right)}{2 \kappa}, 1+\frac{i\left(\omega^{\prime}-\omega\right)}{2 \kappa} ; 2 ; 1-\frac{k^{2}}{k^{\prime 2}}\right) \\
& +\frac{i \tilde{f}\left(\tilde{\mathbf{k}}_{+}\right) e^{-i\left(\omega+\omega^{\prime}\right) v_{0}} \sqrt{\sinh (\pi \omega / \kappa) \sinh \left(\pi \omega^{\prime} / \kappa\right)}\left(\frac{k}{\sqrt{2} \kappa}\right)^{-i \omega / \kappa}\left(\frac{k^{\prime}}{\sqrt{2} \kappa}\right)^{-i \omega^{\prime} / \kappa}}{} \\
& \times \Gamma\left(1+\frac{i \omega}{\kappa}\right) \Gamma\left(1+\frac{i \omega^{\prime}}{\kappa}\right) \Gamma\left(1+\frac{i \omega}{\kappa}+\frac{i \omega^{\prime}}{\kappa}\right) \\
& \times\left[\frac{\tilde{k}_{+}^{2}}{k^{2}}\left(1+\frac{i \omega^{\prime}}{\kappa}\right){ }_{2} F_{1}\left(2,1+\frac{i \omega}{\kappa} ; 3+\frac{i \omega}{\kappa}+\frac{i \omega^{\prime}}{\kappa} ; 1-\left(\frac{k^{\prime}}{k}\right)^{2}\right)\right. \\
& \left.+\frac{4 k_{A} \tilde{k}_{+}^{A}}{\left(k^{\prime}\right)^{2}}\left(1+\frac{i \omega}{\kappa}\right)_{2} F_{1}\left(2,1+\frac{i \omega^{\prime}}{\kappa} ; 3+\frac{i \omega}{\kappa}+\frac{i \omega^{\prime}}{\kappa} ; 1-\left(\frac{k}{k^{\prime}}\right)^{2}\right)\right]
\end{aligned}
$$

where $\tilde{\mathbf{k}}=\mathbf{k}-\mathbf{k}^{\prime}, \tilde{\mathbf{k}}_{+}=\mathbf{k}+\mathbf{k}^{\prime}, \tilde{f}$ is the Fourier transform of $f$ as defined by

$$
\tilde{f}(\mathbf{k})=\int e^{i k_{A} x^{A}} f(x, y) d x d y,
$$

and $P$ stands for the Cauchy principal value.

We see that both $\alpha_{\omega, \mathbf{k} ; \omega^{\prime}, \mathbf{k}^{\prime}}^{(1)}$ and $\beta_{\omega, \mathbf{k} ; \omega^{\prime}, \mathbf{k}^{\prime}}^{(1)}$ are nonvanishing for generic $f . \alpha_{\omega, \mathbf{k} ; \omega^{\prime}, \mathbf{k}^{\prime}}^{(1)}$ is distributional at $\omega=\omega^{\prime}$, having both a Dirac delta and a Cauchy principal value there, whereas $\beta_{\omega, \mathbf{k} ; \omega^{\prime}, \mathbf{k}^{\prime}}^{(1)}$ has no distributional singularities.

\section{B. Quantized field}

We are now ready to read off the quantum memory associated with the shell.

Since the modes $\phi_{\omega, \mathbf{k}}^{0}$ are of positive frequency with respect to the Killing vector $\partial_{v}$ for $v<v_{0}$, and the modes $\phi_{\omega, \mathbf{k}}^{1}$ are of positive frequency with respect to the Killing vector $\partial_{v}$ for $v>v_{0}$, we can quantize the field in each region by adopting these modes as the positive frequency basis functions. As $\partial_{v}$ generates a pure boost in each region, the Fock vacua that ensue are of the Rindler type, seen as a no-particle state by the uniformly accelerated observers who follow the orbits of $\partial_{v}$.

However, the Bogoliubov transformation (3.14) between the two sets of modes is nontrivial, and in particular it involves nonvanishing beta-coefficients. It follows that the two Rindler vacua are not equivalent: if the field is initially prepared in the $v<v_{0}$ Rindler vacuum, the field is no longer in the Rindler vacuum for $v>v_{0}$.

Hence, the shell creates Rindler particles that contain information about the classical supertranslation field $\Xi$, and specifically about the planar profile $f$ of the supertranslational shockwave. This is a quantum counterpart of the classical Rindler supertranslation memory found in [24].
In particular, if the field is prepared in the Minkowski vacuum at $v<v_{0}$, the reduced density matrix in the right Rindler wedge will acquire nonthermal corrections for $v>v_{0}$. The extra Rindler particles created by the shell change the entanglement between observers who reside in the opposite Rindler wedges. We shall analyze this phenomenon in the next section.

\section{ENTANGLEMENT DUE TO RINDLER SUPERTRANSLATIONS}

\section{A. The entanglement setup}

It is well appreciated that Minkowski vacuum contains nonlocal spatial correlations that can be harvested by localized quantum systems [29-32]. For a pair of localized observers who follow the orbits of a boost Killing vector, accelerating in opposite directions with acceleration of magnitude $a$, these quantum correlations appear as a twomode squeezed state, and each of the individual observers experiences the state as thermal in the Unruh temperature $a /(2 \pi)[15]$.

Suppose now that one of the accelerated observers goes through the shock wave (2.5). How does the shock wave affect the quantum correlations between the two observers?

To set up the notation, we call the two observers respectively Luke and Rob, with Luke accelerating to the left and Rob accelerating to the right, as shown in Fig. 2. In the past of the shell, the Minkowski vacuum $|0\rangle_{M}$ can be written as [16]

$$
|0\rangle_{M}=\prod_{\omega, \mathbf{k}} \sqrt{1-e^{-2 \pi \omega / \kappa}} \sum_{n=0}^{\infty} e^{-n \pi \omega / \kappa}|n\rangle_{L, \omega, \mathbf{k}} \otimes|n\rangle_{R, \omega, \mathbf{k}},
$$

where $|n\rangle_{R, \omega, \mathbf{k}}$ are the Fock basis states in region I in the notation of Sec. III and $|n\rangle_{L, \omega, \mathbf{k}}$ are the corresponding Fock 


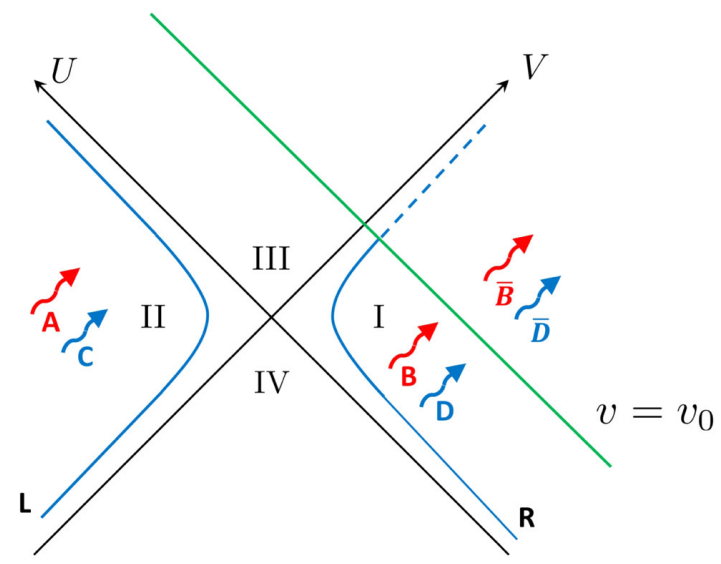

FIG. 2. A pair of uniformly accelerated observers in the shock wave spacetime of Fig. 1. Luke (L) accelerates leftward in quadrant II. Rob (R) accelerates rightward in quadrant I, crossing the wave. After crossing the wave, Rob's trajectory is no longer a pure hyperbola in the two dimensions shown in the diagram, but it involves a perturbative correction due to the wave [24]. Luke couples to field modes labeled $A$ and $C$. Rob couples before the wave-crossing to field modes labeled $B$ and $D$, and after the wave-crossing to field modes labelled $\bar{B}$ and $\bar{D}$.

basis states in region II. If the observers' proper acceleration has magnitude $a$, the frequency with respect to the observers' proper time is related to $\omega$ by $(a / \kappa) \omega$.

To describe the correlations between Luke and Rob after Rob has crossed the shock wave, we need to write (4.1) in Rob's new basis, obtained from the old basis by the Bogoliubov transformation (3.15).

We simplify this problem in two ways. First, instead of the continuous labels $\omega$ and $\mathbf{k}$, we postulate that Rob and Luke each couple to just two modes of the field. This sidesteps the technical issue that the product over the modes on the right-hand side of (4.1) is not mathematically well defined, and the related open questions of quantifying entanglement with continuously labeled mode sets. Conditions under which this postulate may provide a reasonable approximation in a sense of wave packets are discussed in [33]. Second, we truncate the initial state of each mode to keep just the $n=0$ and $n=1$ states. From (4.1) we see that is a good approximation for the high energy modes, $\omega / \kappa \gg 1$.

\section{B. Before the wave}

We denote the two modes to which Luke couples by $A$ and $C$, and the two modes to which Rob couples by $B$ and $D$. Before the shock wave, we take the state to be

$$
|\Phi\rangle=\left|\phi_{1}\right\rangle \otimes\left|\phi_{2}\right\rangle
$$

where

$$
\begin{aligned}
& \left|\phi_{1}\right\rangle=\frac{1}{\sqrt{1+p^{2}}}\left(|0\rangle_{A} \otimes|0\rangle_{B}+p|1\rangle_{A} \otimes|1\rangle_{B}\right), \\
& \left|\phi_{2}\right\rangle=\frac{1}{\sqrt{1+q^{2}}}\left(|0\rangle_{C} \otimes|0\rangle_{D}+q|1\rangle_{C} \otimes|1\rangle_{D}\right),
\end{aligned}
$$

and $p$ and $q$ are real-valued parameters. $|\Phi\rangle$ is a good approximation to the high-frequency regime in (4.1) when $0<p \ll 1$ and $0<q \ll 1$, but in what follows we consider the more general situation in which $p$ and $q$ are allowed to be arbitrary.

We quantify the entanglement in $|\Phi\rangle$ by the negativity $\mathcal{N}$, reviewed in Appendix B. $\left|\phi_{1}\right\rangle$ is bipartite in $A \leftrightarrow B$ and has negativity $p /\left(1+p^{2}\right)$; similarly, $\left|\phi_{2}\right\rangle$ is bipartite in $C \leftrightarrow D$ and has negativity $q /\left(1+q^{2}\right)$. There is clearly no entanglement in the subsystems $A \leftrightarrow C, A \leftrightarrow D, B \leftrightarrow D$ and $B \leftrightarrow C$, and the corresponding negativities vanish. Collecting, the nonvanishing negativities are

$$
\begin{aligned}
& \mathcal{N}_{A \leftrightarrow B}=\frac{p}{1+p^{2}}, \\
& \mathcal{N}_{C \leftrightarrow D}=\frac{q}{1+q^{2}} .
\end{aligned}
$$

The total Rob-Luke negativity is $\mathcal{N}_{A \leftrightarrow B}+\mathcal{N}_{C \leftrightarrow D}=$ $p /\left(1+p^{2}\right)+q /\left(1+q^{2}\right)$.

\section{After the wave}

After Rob has crossed the wave, we denote the two modes to which Rob couples by $\bar{B}$ and $\bar{D}$. We write the Bogoliubov coefficients from $\{B, D\}$ to $\{\bar{B}, \bar{D}\}$ as in (3.14) and (3.15),

$$
\begin{aligned}
& \alpha_{k, k^{\prime}}=\delta_{k, k^{\prime}}+\lambda \alpha_{k, k^{\prime}}^{(1)}+\lambda^{2} \alpha_{k, k^{\prime}}^{(2)}+\mathcal{O}\left(\lambda^{3}\right), \\
& \beta_{k, k^{\prime}}=\lambda \beta_{k, k^{\prime}}^{(1)}+\lambda^{2} \beta_{k, k^{\prime}}^{(2)}+\mathcal{O}\left(\lambda^{3}\right),
\end{aligned}
$$

where $k \in\{B, D\}$ and $k^{\prime} \in\{\bar{B}, \bar{D}\}$, and we have included a formal perturbative parameter $\lambda$ to facilitate the bookkeeping in the perturbative expansion.

For (4.5) to provide a mathematically consistent Bogoliubov transformation, $\alpha_{k, k^{\prime}}$ and $\beta_{k, k^{\prime}}$ must satisfy the Bogoliubov identities [16], which imply in the linear order that $\alpha^{(1)}$ is anti-Hermitian and $\beta^{(1)}$ is symmetric, while in higher orders they imply relations involving the higher-order coefficients (see Appendix A of [34]). Reducing (3.17) to (4.5) in a way that satisfies these identities would need additional input about the reduction, such as a construction of suitable wave packets [33], and we shall not attempt to provide this input here. Instead, we shall proceed without specifying the explicit form of $\alpha_{k, k^{\prime}}^{(1)}$ and $\beta_{k, k^{\prime}}^{(1)}$. This will suffice to demonstrate that the shock 
wave does change the bipartite entanglements in the system.

\section{Changes in entanglement}

In terms of the Bogoliubov transformation (4.5), we have $[16,35]$

$$
\begin{aligned}
a_{k}^{\dagger} & =\sum_{k^{\prime}}\left(\alpha_{k, k^{\prime}} \bar{a}_{k^{\prime}}^{\dagger}-\beta_{k, k^{\prime}} \bar{a}_{k^{\prime}}\right), \\
|0\rangle_{B}|0\rangle_{D} & =N e^{\frac{1}{2} \sum_{m n} V_{m n} \bar{a}_{m}^{\dagger} \bar{a}_{n}^{\dagger}}|0\rangle_{\bar{B}}|0\rangle_{\bar{D}},
\end{aligned}
$$

where $a_{m}$ and $a_{m}^{\dagger}$ are the annihilation and creation operators for Rob's early time modes $B$ and $D, \bar{a}_{m}$ and $\bar{a}_{m}^{\dagger}$ are the annihilation and creation operators for Rob's late time modes $\bar{B}$ and $\bar{D}, V_{m n}=\beta_{m p}^{\dagger}\left(\alpha^{-1}\right)_{p n}^{\dagger}$, and $N$ is a normalization constant. Using (4.6), we can transform the state $|\Phi\rangle$ (4.2) to the late time basis and analyze the negativity for the bipartite subsystems of interest. We shall omit the calculational details and just describe the outcome.

\section{Subsystem $A$ and $\bar{B}$.}

Consider the system formed by $A$ and $\bar{B}$. Before the wave this was the single pair $\left|\phi_{1}\right\rangle$ (4.3a), one mode coupling to Luke and the other to Rob, with negativity $\mathcal{N}_{A \leftrightarrow B}$ (4.4a).

After the wave, the reduced density matrix $\rho_{A \leftrightarrow \bar{B}}$ is obtained by tracing out $C$ and $\bar{D}$. Keeping terms of order $\lambda^{2}$, we find that the partial transpose $\rho_{A \leftrightarrow \bar{B}}^{T}$ is a 12 by 12 matrix, and the correction to $\mathcal{N}_{A \leftrightarrow B}$ (4.4a) starts in order $\lambda^{2}$. We consider this correction here in the limit in which the diagonal elements of $\alpha^{(1)}$ and $\beta^{(1)}$ are negligible compared with the off-diagonal elements; this limit can be motivated by observing that in the continuous label case (3.19), the last two terms in (3.19a) and the first term in (3.19b) vanish on the diagonal. The correction to the negativity comes then entirely from the correction to the single negative eigenvalue of $\rho_{A \leftrightarrow B}^{T}$, and we find

$$
\begin{aligned}
\mathcal{N}_{A \leftrightarrow \bar{B}}= & \frac{p}{1+p^{2}}-\lambda^{2}\left(\frac{p^{2}\left(1+2 q^{2}\right)+q^{2}+p\left(1+5 q^{2}\right)}{2\left(1+p^{2}\right)\left(1+q^{2}\right)}\left|\alpha_{B, D}^{(1)}\right|^{2}+\frac{p\left[p^{2} q^{2}+1+2 q^{2}+p\left(3+5 q^{2}\right)\right]\left(1+q^{2}\right)-2 p^{4} q^{4}}{2 p\left(1+p^{2}\right)\left(1+q^{2}\right)^{2}}\left|\beta_{B, D}^{(1)}\right|^{2}\right) \\
& +\mathcal{O}\left(\lambda^{3}\right) .
\end{aligned}
$$

As both $\alpha^{(1)}$ and $\beta^{(1)}$ appear in (4.7), the change in the entanglement is due in part to particle creation and in part to mode mixing. The sign of the correction term in (4.7) is typically negative, that is, entanglement is degraded. However, it can be arranged to be positive if $\beta_{B, D}^{(1)}$ is nonzero and $p$ and $q$ are sufficiently large. An increase in the entanglement, when it occurs, is hence necessarily associated with particle creation.

For the Minkowski vacuum state (4.1) in the high frequency limit, $\omega / \kappa \gg 1$, the correction term in (4.7) is negative since in this case $0<p \ll 1$ and $0<q \ll 1$. The wave has hence degraded the entanglement between $A$ and $\bar{B}$.

An interesting special case occurs when $q=0$ and $p=1$ : there is then initially only one entangled pair, and this pair is prepared in the maximally entangled Bell state. The initially maximal entanglement is degraded, as seen from the sign of the correction in (4.7). This system is mathematically identical to the cavity system considered in [36], and (4.7) agrees with the correction found therein.

\section{Subsystem $\bar{B}$ and $\bar{D}$}

Consider the system formed by $\bar{B}$ and $\bar{D}$. Before the wave this was a system of two completely unentangled modes coupled to Rob, with vanishing negativity.

After the wave, the reduced density matrix $\rho_{\bar{B} \leftrightarrow \bar{D}}$ is obtained by tracing out Luke's modes $A$ and $C$. Keeping terms of order $\lambda^{2}$, we find that the partial transpose $\rho_{\bar{B} \leftrightarrow \bar{D}}^{T}$ is a 14 by 14 matrix. The leading correction to the negativity appears in order $\lambda$, and we find

$$
\mathcal{N}_{\bar{B} \leftrightarrow \bar{D}}=\lambda \frac{2 p^{2} q^{2}\left|\beta_{B, D}^{(1)}\right|}{\left(1+p^{2}\right)\left(1+q^{2}\right)}+\mathcal{O}\left(\lambda^{2}\right)
$$

The wave has hence entangled Rob's two modes. As the Bogoliubov coefficient entering (4.8) is $\beta^{(1)}$, the leading order entanglement creation is due to particle creation, not due to mode mixing.

\section{Subsystem $\bar{B}$ and $C$}

Consider finally the system formed by $\bar{B}$ and $C$. Before the wave this was a system of two completely unentangled modes, one coupled to Luke and the other to Rob, with vanishing negativity.

After the wave, the reduced density matrix $\rho_{\bar{B} \leftrightarrow C}$ is obtained by tracing out Luke's mode $A$ and Rob's mode $\bar{D}$. Keeping terms of order $\lambda^{2}$, we find that the partial transpose $\rho_{\bar{B} \leftrightarrow C}^{T}$ is a 12 by 12 matrix, and the first contribution to $\mathcal{N}_{\bar{B} \leftrightarrow C}$ comes in order $\lambda^{2}$. Specialising again to the limit in which the diagonal elements of $\alpha^{(1)}$ and $\beta^{(1)}$ are negligible compared with the off-diagonal elements, we find 


$$
\begin{aligned}
\mathcal{N}_{\bar{B} \leftrightarrow C}= & \lambda^{2}\left(2 q^{2} \max \left(2 p^{2} q^{2}\left|\alpha_{B, D}^{(1)}\right|^{2}-\left|\beta_{B, C}^{(1)}\right|^{2}, 0\right)\right. \\
& \left.+p^{2} \max \left(\left(2 q^{2}-1\right)\left|\alpha_{B, D}^{(1)}\right|^{2}+2\left(q^{4}-1\right)\left|\beta_{B, C}^{(1)}\right|^{2}, 0\right)\right) \\
& +\mathcal{O}\left(\lambda^{3}\right) .
\end{aligned}
$$

There exist parameter ranges in which $\mathcal{N}_{\bar{B} \leftrightarrow C}>0$, and the entanglement generation comes from a mixture of particle creation and mode mixing effects.

\section{Quantum monogamy and negativity}

A curious property in the above negativity results is that entanglement generation can in certain circumstances happen already in order $\lambda$, as seen in (4.8), but entanglement degradation will happen only in order $\lambda^{2}$, as seen in (4.7). For example, suppose that $p=1$, and consider the entanglement of $\bar{B}$ with $A$ and with $\bar{D} . \mathcal{N}_{A \leftrightarrow \bar{B}}$ (4.7) has decreased from the maximal entanglement value $1 / 2$ in order $\lambda^{2}$, but $\mathcal{N}_{\bar{B} \leftrightarrow \bar{D}}$ (4.8) has increased from the vanishing entanglement value 0 already in order $\lambda$. This might at first sight appear to be at tension with the monogamy of entanglement, which states that given a pair of maximally entangled systems, neither member of the pair can be entangled with a third system [37].

However, there is in fact no tension. The reason is that the monogamy inequality that relates to negativity is not linear but quadratic, taking in the present situation the form [38] (for related discussion see [39-41])

$$
\mathcal{N}_{\bar{B} \leftrightarrow A C \bar{D}}^{2} \geq \mathcal{N}_{A \leftrightarrow \bar{B}}^{2}+\mathcal{N}_{\bar{B} \leftrightarrow C}^{2}+\mathcal{N}_{\bar{B} \leftrightarrow \bar{D}}^{2} .
$$

An explicit calculation of the entanglement of $\bar{B}$ with $A, C$, and $\bar{D}$ (negative eigenvalues of the $16 \times 16$ matrix obtained by taking the partial transpose of late time $\rho=|\Phi\rangle\langle\Phi|$ with respect to $\bar{B}$ ) shows that $\mathcal{N}_{\bar{B} \leftrightarrow A C \bar{D}}$ does not obtain a correction at order $\lambda$ from the maximal entanglement value of $1 / 2$, for the maximal case when $p=1$ and $q=1$, but gets possible corrections starting from order $\lambda^{2}$. Hence, none of the terms on either side of the inequality (4.10) are linear in $\lambda$, since the only linear order term generated in $\mathcal{N}_{\bar{B} \leftrightarrow \bar{D}}$ in (4.8) becomes order $\lambda^{2}$ upon squaring on the right-hand side. Inequality (4.10) is thus satisfied to order $\lambda$ and there is no contradiction.

From our results in (4.7), (4.8), and (4.9), it is straightforward to check that the $\lambda^{2}$ correction term on the righthand side of (4.10) for the maximal case when $p=1$ and $q=1$ is given by

$$
\begin{aligned}
\mathcal{N}_{A \leftrightarrow \bar{B}}^{2}+\mathcal{N}_{\bar{B} \leftrightarrow C}^{2}+\mathcal{N}_{\bar{B} \leftrightarrow \bar{D}}^{2}= & \frac{1}{4}-\frac{5}{4}\left(\left|\alpha_{B, D}^{(1)}\right|^{2}+2\left|\beta_{B, D}^{(1)}\right|^{2}\right) \lambda^{2} \\
& +\mathcal{O}\left(\lambda^{3}\right) .
\end{aligned}
$$

As is expected, the coefficient of the $\lambda^{2}$ term is nonpositive. For the left-hand side of (4.10), to obtain the explicit form of the $\lambda^{2}$ term in $\mathcal{N}_{\bar{B} \leftrightarrow A C \bar{D}}^{2}$, one arrives at a $81 \times 81$ partially transposed matrix with respect $\bar{B}$. Calculating the eigenvalues of such a large matrix is highly nontrivial computationally and beyond the scope of the present work. However, we refer the reader to [38-41] wherein a detailed discussion of the above monogamy inequality for negativity can be found.

\section{DISCUSSION}

We have shown that a classical supertranslation hair implanted on a Rindler horizon by a shock wave induces in quantum field theory a quantum supertranslation memory that modulates the entanglement between the two opposing Rindler wedges. In the Bogoliubov coefficient description, this memory involves nontrivial alpha-coefficients and nontrivial beta-coefficients, so that there is both particle creation and mode mixing. Within an entanglement analysis that truncates the number of field modes, we identified subsystems whose entanglement is degraded and subsystems whose entanglement is enhanced, and the entanglement effect appears to be robust against the input used in the truncation. Similar entanglement degradation and generation has been previously found in cavity systems in noninertial motion $[36,42,43]$.

The linearized stress-energy tensor of the supertranslated metric (2.5) is given in equation (2.9) of [24]. This stressenergy tensor is linear in the function $f$ that characterizes the planar asymmetry of the shock wave, and for a generic $f$ the stress-energy tensor breaks the usual energy conditions somewhere, in particular on crossing the Rindler horizon from region I to region III in Fig. 1. Further, for a given $f$, these violations become arbitrarily large near $\mathcal{I}^{-}$: this phenomenon stems from the diverging norm of the Rindler Killing vector $\partial_{v}$ near the infinity, and indicates that linearized perturbation theory is not reliable to arbitrarily large $r$. However, it is possible to amend the shock wave by adding to $T_{v v}$ a uniform surface energy density $\mu$, as shown in Eq. (2.10) of [24], and for a wave released at any finite value of $r$. The amended stress-energy tensor may still break the energy condition due to quantum effects, however we note that the time averaged quantity, $\int_{v_{0}-\epsilon}^{v_{0}+\epsilon} d v T_{v v}$ then satisfies the null energy condition provided $\mu$ is chosen sufficiently large. This is similar to the shock wave in the Schwarzschild black hole case considered in [2], where a sufficiently large $\mu$ makes the null energy condition hold everywhere except possibly near the singularity where the linearized theory becomes unreliable. Within our linearized treatment, the Bogoliubov coefficients for the amended supertranslated wave in Rindler would contain a new additive term coming from $\mu$. The explicit form of the correction would need to be determined by an analysis similar to that in Sec. III.

We anticipate that a similar analysis can be carried out for a shock wave that implants supertranslations on a Schwarzschild black hole [2], as shown in Fig. 3, leading to 


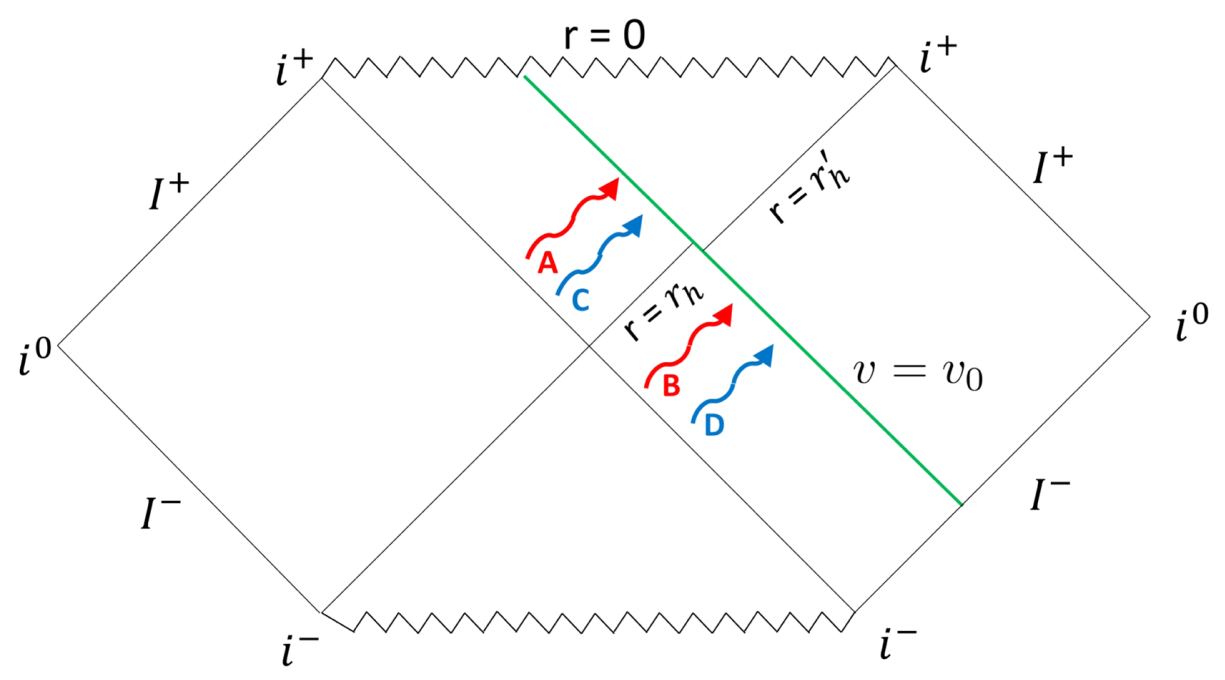

FIG. 3. The extended Schwarzschild spacetime with an infalling, supertranslation-implanting shock wave at $v=v_{0}$ [2]. Two Hawking pairs are shown, $A \leftrightarrow B$ (red) and $C \leftrightarrow D$ (blue).

nontrivial Bogoliubov coefficients in the region $v>v_{0}$ outside the black hole. There are now pairs of Hawking quanta created near the horizon, depicted as the pair $A \leftrightarrow B$ and the pair $C \leftrightarrow D$ in the figure, such that $A$ and $C$ are behind the Killing horizon while $B$ and $D$ are outgoing modes which an asymptotic observer at infinity will eventually detect as Hawking radiation. Each of the pairs $A \leftrightarrow B$ and $C \leftrightarrow D$ is maximally entangled. The infalling shock wave will then affect the entanglement between the interior quanta and the escaping quanta very much as in our Rindler analysis, so that the shock wave imprints its information on the Hawking quanta as a quantum memory. This may counteract the conventional argument that any characteristic information about infalling matter or radiation is lost in the Hawking evaporation $[44,45]$, and it may have a role in the proposal that supertranslations provide a solution to the black hole information paradox [1-3] and in establishing a quantum version of black hole hair theorems.

\section{ACKNOWLEDGMENTS}

We thank Gerardo Adesso and Bartosz Regula for a helpful discussion on entanglement monotones and Masahiro Hotta for bringing the work in $[5,6]$ to our attention. S. K. thanks the University of Nottingham and CPT, Aix Marseille Université, Université de Toulon,
CNRS for hospitality and the Department of Science and Technology, India, for partial financial support. J. L. was supported in part by the Science and Technology Facilities Council (Theory Consolidated Grants No. ST/J000388/1 and No. ST/P000703/1).

\section{APPENDIX A: BESSEL INTEGRA IDENTITY}

The evaluation of the Bogoliubov coefficients in Sec. III uses the identity

$$
\begin{aligned}
& \int_{0}^{\infty} \frac{d x}{x} K_{i \Omega}(a x) K_{i \Omega^{\prime}}(b x) \\
& =\frac{\pi^{2}(b / a)^{i \Omega}}{2 \Omega \sinh (\pi \Omega)}\left[1+\frac{i q \Omega}{2}{ }_{2} F_{1}(1+i \Omega, 1 ; 2 ; q)\right] \delta\left(\Omega-\Omega^{\prime}\right) \\
& \quad+\frac{\pi^{2} q(b / a)^{i \Omega^{\prime}}}{8}{ }_{2} F_{1}\left(1+\frac{i\left(\Omega+\Omega^{\prime}\right)}{2}, 1+\frac{i\left(\Omega^{\prime}-\Omega\right)}{2} ; 2 ; q\right) \\
& \quad \times \frac{1}{\sinh \left(\pi\left(\Omega+\Omega^{\prime}\right) / 2\right)} P\left(\frac{1}{\sinh \left(\pi\left(\Omega-\Omega^{\prime}\right) / 2\right)}\right),
\end{aligned}
$$

where $a>0, b>0, \Omega>0, \Omega^{\prime}>0, q=1-b^{2} / a^{2},{ }_{2} F_{1}$ is the Gaussian hypergeometric function [25] and $P$ denotes the Cauchy principal value.

To verify (A.1), let $\epsilon>0$. We then have

$$
\begin{aligned}
\int_{0}^{\infty} \frac{d x}{x^{1-\epsilon}} K_{i \Omega}(a x) K_{i \Omega^{\prime}}(b x)= & \frac{(b / a)^{i \Omega^{\prime}} a^{-\epsilon}}{2^{3-\epsilon} \Gamma(\epsilon)}\left|\Gamma\left(\frac{\epsilon+i\left(\Omega+\Omega^{\prime}\right)}{2}\right) \Gamma\left(\frac{\epsilon+i\left(\Omega-\Omega^{\prime}\right)}{2}\right)\right|_{2}^{2} F_{1}\left(\frac{\epsilon+i\left(\Omega+\Omega^{\prime}\right)}{2}, \frac{\epsilon+i\left(\Omega^{\prime}-\Omega\right)}{2} ; \epsilon ; q\right) \\
= & \frac{Q_{\epsilon} \pi^{2}(b / a)^{i \Omega^{\prime}}\left(\Omega-\Omega^{\prime}\right)}{2\left(\Omega+\Omega^{\prime}\right) \sinh \left(\pi\left(\Omega+\Omega^{\prime}\right) / 2\right) \sinh \left(\pi\left(\Omega-\Omega^{\prime}\right) / 2\right)} \\
& \times \frac{\epsilon}{\left(\Omega-\Omega^{\prime}\right)^{2}+\epsilon^{2}}{ }_{2} F_{1}\left(\frac{\epsilon+i\left(\Omega+\Omega^{\prime}\right)}{2}, \frac{\epsilon+i\left(\Omega^{\prime}-\Omega\right)}{2} ; \epsilon ; q\right)
\end{aligned}
$$


where $Q_{\epsilon}$ has the property that $Q_{\epsilon} \rightarrow 1$ as $\epsilon \rightarrow 0$. The first equality in (A.2) follows from formula 6.576.4 in [28], and the second equality follows using standard properties of the Gamma-function [25].

To evaluate the $\epsilon \rightarrow 0$ limit in (A.2), we expand ${ }_{2} F_{1}$ in its power series [25] and use in each term the distributional identity

$$
\lim _{\epsilon \rightarrow 0_{+}} \frac{1}{x \pm i \epsilon}=P\left(\frac{1}{x}\right) \mp i \pi \delta(x)
$$

with the outcome (A.1).

\section{APPENDIX B: NEGATIVITY}

For all of our bipartite quantum systems, we quantify the entanglement by the negativity, defined by [46-48]

$$
\mathcal{N}=\frac{1}{2}\left(\left\|\rho^{T}\right\|-1\right),
$$

where $\rho$ is the density matrix, the superscript $T$ denotes the partial transpose, that is, the transpose in one of the subsystems, and $\|\cdot\|$ is the trace norm. An equivalent formula is

$$
\mathcal{N}=\sum_{i} \frac{1}{2}\left(\left|\lambda_{i}\right|-\lambda_{i}\right)
$$

where $\lambda_{i}$ are the eigenvalues of $\rho^{T}$.

$\mathcal{N}$ is non-negative, and a strictly positive value of $\mathcal{N}$ implies that the system is not separable. $\mathcal{N}$ does not in general coincide with the entanglement entropy, but it is an entanglement monotone, and although its operational meaning is subtle [49,50], it provides a convenient interpolation between other entanglement monotones with a more direct operational meaning [51].

The main advantage of $\mathcal{N}$ is that it is easy to compute in systems of arbitrary dimension. In this paper we consider applications to finite-dimensional Hilbert spaces; however, $\mathcal{N}$ generalizes to infinite-dimensional Hilbert spaces, and it has a particularly convenient form for Fock state spaces in the continuous-variable formalism [52,53].
[1] S. W. Hawking, M. J. Perry, and A. Strominger, Soft Hair on Black Holes, Phys. Rev. Lett. 116, 231301 (2016).

[2] S. W. Hawking, M. J. Perry, and A. Strominger, Superrotation charge and supertranslation hair on black holes, J. High Energy Phys. 05 (2017) 161.

[3] A. Strominger, Black Hole Information Revisited, arXiv:1706.07143 [Phys. Rev. Lett. (to be published)].

[4] D. Kapec, M. Perry, A. M. Raclariu, and A. Strominger, Infrared divergences in QED, revisited, Phys. Rev. D 96, 085002 (2017).

[5] M. Hotta, K. Sasaki, and T. Sasaki, Diffeomorphism on horizon as an asymptotic isometry of Schwarzschild black hole, Classical Quantum Gravity 18, 1823 (2001).

[6] M. Hotta, Holographic charge excitations on horizontal boundary, Phys. Rev. D 66, 124021 (2002).

[7] A. Strominger, On BMS invariance of gravitational scattering, J. High Energy Phys. 07 (2014) 152.

[8] J. Winicour, Global aspects of radiation memory, Classical Quantum Gravity 31, 205003 (2014).

[9] A. Ashtekar, Geometry and physics of null infinity, Surveys in Differential Geometry 20, 99 (2015).

[10] A. Strominger and A. Zhiboedov, Gravitational memory, BMS supertranslations and soft theorems, J. High Energy Phys. 01 (2016) 086.

[11] M. Mirbabayi and M. Porrati, Dressed Hard States and Black Hole Soft Hair, Phys. Rev. Lett. 117, 211301 (2016).

[12] R. Bousso and M. Porrati, Observable supertranslations, Phys. Rev. D 96, 086016 (2017).
[13] S. A. Fulling, Nonuniqueness of canonical field quantization in Riemannian space-time, Phys. Rev. D 7, 2850 (1973).

[14] P. C. W. Davies, Scalar particle production in Schwarzschild and Rindler metrics, J. Phys. A 8, 609 (1975).

[15] W. G. Unruh, Notes on black hole evaporation, Phys. Rev. D 14, 870 (1976).

[16] N. D. Birrell and P.C.W. Davies, Quantum Fields in Curved Space (Cambridge University Press, Cambridge, England, 1982).

[17] R. M. Wald, Quantum Field Theory in Curved Spacetime and Black Hole Thermodynamics (University of Chicago Press, Chicago, 1994).

[18] B. Zeldovich and A. G. Polnarev, Radiation of gravitational waves by a cluster of superdense stars, Sov. Astron. Lett. 18, 17 (1974).

[19] L. Donnay, G. Giribet, H. A. Gonzalez, and M. Pino, Supertranslations and Superrotations at the Black Hole Horizon, Phys. Rev. Lett. 116, 091101 (2016).

[20] L. Donnay, G. Giribet, H. A. Gonzlez, and M. Pino, Extended symmetries at the black hole horizon, J. High Energy Phys. 09 (2016) 100.

[21] C. Eling and Y. Oz, On the membrane paradigm and spontaneous breaking of horizon BMS symmetries, J. High Energy Phys. 07 (2016) 065.

[22] R. G. Cai, S. M. Ruan, and Y. L. Zhang, Horizon supertranslation and degenerate black hole solutions, J. High Energy Phys. 09 (2016) 163. 
[23] M. Hotta, J. Trevison, and K. Yamaguchi, Gravitational memory charges of supertranslation and superrotation on Rindler horizons, Phys. Rev. D 94, 083001 (2016).

[24] S. Kolekar and J. Louko, Gravitational memory for uniformly accelerated observers, Phys. Rev. D 96, 024054 (2017).

[25] NIST Digital Library of Mathematical Functions, http:// dlmf.nist.gov/, Release 1.0.15 of 2017-06-01, edited by F. W. J. Olver, A. B. Olde Daalhuis, D. W. Lozier, B. I. Schneider, R. F. Boisvert, C. W. Clark, B. R. Miller, and B. V. Saunders.

[26] G. W. Gibbons, Quantized fields propagating in plane wave space-times, Commun. Math. Phys. 45, 191 (1975).

[27] J. Garriga and E. Verdaguer, Scattering of quantum particles by gravitational plane waves, Phys. Rev. D 43, 391 (1991).

[28] I. S. Gradshteyn and I. M. Ryzhik, Table of Integrals, Series, and Products, 7th ed. (Academic Press, New York, 2007).

[29] A. Valentini, Non-local correlations in quantum electrodynamics, Phys. Lett. 153A, 321 (1991).

[30] B. Reznik, Entanglement from the vacuum, Found. Phys. 33, 167 (2003).

[31] B. Reznik, A. Retzker, and J. Silman, Violating Bell's inequalities in the vacuum, Phys. Rev. A 71, 042104 (2005).

[32] A. Pozas-Kerstjens and E. Martín-Martínez, Harvesting correlations from the quantum vacuum, Phys. Rev. D 92, 064042 (2015).

[33] D. E. Bruschi, J. Louko, E. Martín-Martínez, A. Dragan, and I. Fuentes, The Unruh effect in quantum information beyond the single-mode approximation, Phys. Rev. A 82, 042332 (2010).

[34] J. Doukas and J. Louko, Superconducting circuit boundary conditions beyond the dynamical Casimir effect, Phys. Rev. D 91, 044010 (2015).

[35] A. Fabbri and J. Navarro-Salas, Modeling Black Hole Evaporation (Imperial College Press, London, 2005).

[36] D. E. Bruschi, I. Fuentes, and J. Louko, Voyage to Alpha Centauri: Entanglement degradation of cavity modes due to motion, Phys. Rev. D 85, 061701 (2012).

[37] V. Coffman, J. Kundu, and W. K. Wootters, Distributed entanglement, Phys. Rev. A 61, 052306 (2000).

[38] S. Karmakar, A. Sen, A. Bhar, and D. Sarkar, Strong monogamy conjecture in a four-qubit system, Phys. Rev. A 93, 012327 (2016).
[39] B. Regula, S. Di Martino, S. Lee, and G. Adesso, Strong Monogamy Conjecture for Multiqubit Entanglement: The Four-Qubit Case, Phys. Rev. Lett. 113, 110501 (2014).

[40] J. Choi and J. Kim, Negativity and strong monogamy of multiparty quantum entanglement beyond qubits, Phys. Rev. A 92, 042307 (2015).

[41] B. Regula, A. Osterloh, and G. Adesso, Strong monogamy inequalities for four qubits, Phys. Rev. A 93, 052338 (2016).

[42] N. Friis, D. E. Bruschi, J. Louko, and I. Fuentes, Motion generates entanglement, Phys. Rev. D 85, 081701 (2012).

[43] D. E. Bruschi, J. Louko, D. Faccio, and I. Fuentes, Modemixing quantum gates and entanglement without particle creation in periodically accelerated cavities, New J. Phys. 15, 073052 (2013).

[44] D. Marolf, The black hole information problem: Past, present, and future, Rep. Prog. Phys. 80, 092001 (2017).

[45] W. G. Unruh and R. M. Wald, Information loss, Rep. Prog. Phys. 80, 092002 (2017).

[46] A. Peres, Separability Criterion for Density Matrices, Phys. Rev. Lett. 77, 1413 (1996).

[47] M. Horodecki, P. Horodecki, and R. Horodecki, On the necessary and sufficient conditions for separability of mixed quantum states, Phys. Lett. A 223, 1 (1996).

[48] G. Vidal and R.F. Werner, A computable measure of entanglement, Phys. Rev. A 65, 032314 (2002).

[49] M. B. Plenio, Logarithmic Negativity: A Full Entanglement Monotone That is not Convex, Phys. Rev. Lett. 95, 090503 (2005).

[50] K. Audenaert, M. B. Plenio, and J. Eisert, The Entanglement Cost under Operations Preserving the Positivity of Partial Transpose, Phys. Rev. Lett. 90, 027901 (2003).

[51] M. B. Plenio and S. Virmani, An introduction to entanglement measures, Quantum Inf. Comput. 7, 1 (2007); M. B. Plenio and S. Virmani, Spin chains and channels with memory, Phys. Rev. Lett. 99, 120504 (2007).

[52] R. Simon, Peres-Horodecki Separability Criterion for Continuous Variable Systems, Phys. Rev. Lett. 84, 2726 (2000).

[53] G. Adesso and F. Illuminati, Entanglement in continuous variable systems: Recent advances and current perspectives, J. Phys. A 40, 7821 (2007). 